\title{
SUSY Predictions and SUSY Tools at the LHC
}

\author{
B.C. Allanach*, \\ *DAMTP, CMS, University of Cambridge, Wilberforce road, Cambridge, CB3 0WA, \\ United Kingdom
}

\begin{abstract}
We provide a bestiary of public codes and other algorithmic tools that can be used for analysing supersymmetric phenomenology. We also describe the organisation of the different tools and communication between them. Tools exist that calculate supersymmetric spectra and decay widths, simulate Monte Carlo events as well as those that make predictions of dark matter relic density or that predict precision electroweak or $b$ observables. Some global fitting tools for use in SUSY phenomenology are also presented. In each case, a description and a link to the relevant web-site is provided. It is hoped that this review could serve as an "entry-gate" and map for prospective users.
\end{abstract}




\section{Contents}

1. Introduction

2. Spectrum and Decays 1

2.1 MSSM Sparticle Decays 6

2.2 Higgs Masses and Decays

2.3 NMSSM

3. Matrix Element Generators and Cross Section Calculators 7

4. Event Generators 8

5. Predictions of SUSY Dark Matter 11

6. Predictions for constraints

$6.1 b$ observables 13

6.2 Anomalous magnetic moment of the muon 14

6.3 Electric dipole moments 14

6.4 Electroweak observables 14

7. Fitting tools 15

7.1 Algorithms for multi-dimensional fits

7.2 Example Global Fits to mSUGRA 19

7.3 Data archival 20

8. Summary 21

\section{Introduction}

Analysis in high energy particle physics is becoming increasingly complex; the higher energies and luminosities of current-day colliders lead to higher multiplicities in events. The current high-energy frontier is dominated by hadron-hadron colliders, at the Tevatron ( $p \bar{p}$ at $2 \mathrm{TeV}$ ) or, in the near future, the Large Hadron Collider ( $p p$ at 10 or $14 \mathrm{TeV}$ ), leading to additional complications in describing hadronic initial states and radiation. On the theoretical side, the currently most popular solution to the technical hierarchy problem is the Minimal Supersymmetric Standard Model (MSSM). A low energy parametrisation of the MSSM contains over 100 parameters. In fact, a truly supersymmetric version of the Standard Model contains one less parameter than the Standard Model, since the quartic 


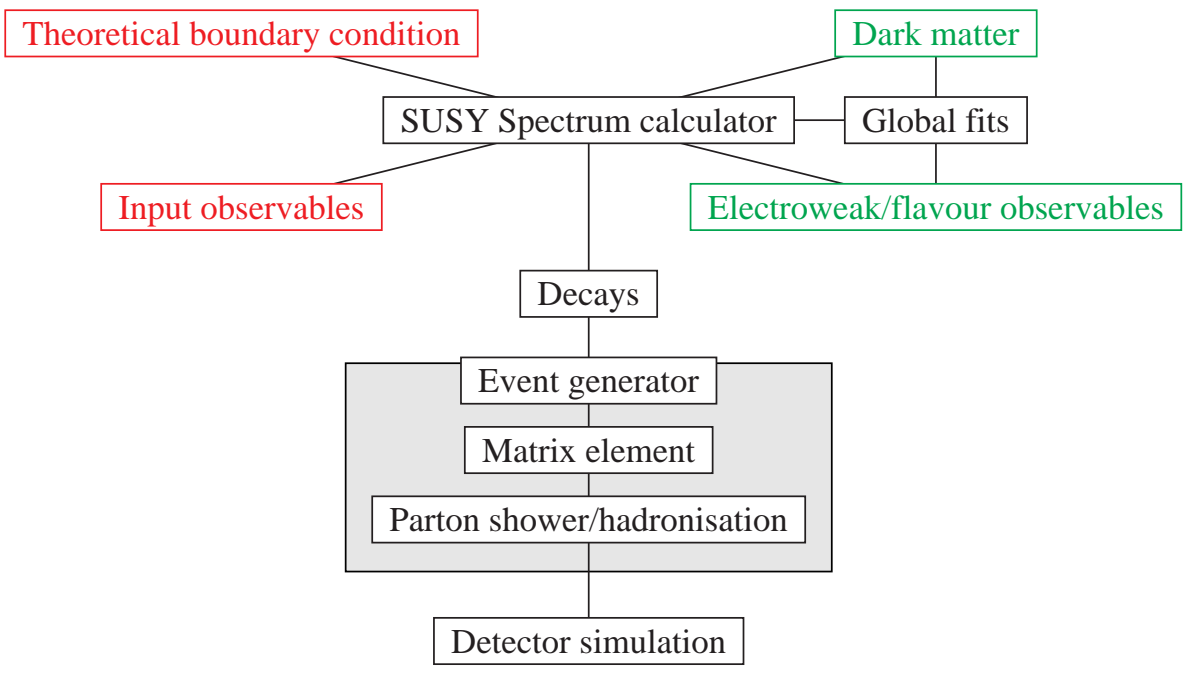

Figure 1: Schematic of the interaction between various programs that perform different SUSY phenomenology calculations. The need for information exchange is denoted by a line.

Higgs coupling becomes a function of the electroweak gauge couplings in the supersymmetric version, instead of being a free parameter. However, in order for the MSSM to be phenomenologically viable, supersymmetry (SUSY) must be broken, and it is in the SUSY breaking sector of the model that the majority of the free parameters lie. The vast majority of this $100+$ dimensional parameter space is ruled out by fairly tight constraints on flavour changing neutral currents. This is often taken to be evidence of some additional structure of the model in the flavour sector. High energy boundary conditions on the supersymmetry parameters that are flavour universal are popular, and may be motivated by various string (and/or grand-unified theory) models.

A schematic of SUSY phenomenology calculations is shown in Fig. 1. Typically, one may want to assume some high energy theoretical boundary condition upon the SUSY breaking sector. One wishes to calculate the MSSM spectrum and couplings consistent with this boundary condition and some input observables $\left(M_{Z}, m_{t} \ldots\right)$ with a spectrum calculator. The spectrum and couplings can then be passed to another program that calculates decays of the various sparticles. Once the masses and decays of the sparticles are calculated, this information may be passed to an event generator in order to randomly simulate several events in some high energy collision. This process is often split into two sub-steps: one performing the hard $2 \rightarrow N$ particle collision (matrix element generation), and one performing hadronic showering, initial state radiation and decays of the sparticles (event generation). Experimental colleagues often then want to pass such simulated events through a detector simulation in order to see how the detector might smear the kinematics. Alternatively, the spectrum and coupling information could be passed to packages which calculate indirect observables, such as the dark matter relic density left in the universe, dark matter direct detection cross-sections, electroweak or $b$-observables. These data may then be used in global fits to the particular SUSY breaking scenario assumed. Some of the programs available perform several of these tasks, but there is currently no single 
program that performs all of the tasks. Previously, information was passed around on an ad hoc basis: each spectrum generator had to be interfaced separately with each program that used its output. With $N$ independent codes, the required number of interfaces such that they could each exchange information to the others was $\sim N$ !. For this reason, several accords have been written and agreed upon in order to cut down on the total number of required interfaces, with an associated reduction in the number of mistakes in the interfacing procedure.

The SUSY Les Houches Accord (SLHA) [1] allows information on the masses and decays of SUSY (and some relevant Standard Model) particles to be passed in between codes. The accord is based on ASCII text, in order to allow easy cross-language communication without introducing platform dependence. The parsing of (files or memory variables containing) such ASCII text is an easy task for many human beings, but the disadvantage of an ASCII format is that developers of tools must write parsing code. Luckily, even this task has been performed, with a SLHA-file parser available [2]. The original SLHA dealt purely with the "vanilla-MSSM": inter-generational sparticle mixing is not taken into account, R-parity and CP are conserved. The second SLHA [3] generalises the possible MSSM models: R-parity violating, $\mathrm{CP}$ and flavour-violating versions of the MSSM are all specified. In addition, the most popular MSSM extension where a Standard Model singlet chiral superfield is added, the Next-to-Minimal Supersymmetric Standard Model (NMSSM), is covered.

The original Les Houches Accord (LHA) 《], allows hard parton-level events to be passed from matrix element calculators onward down the chain to the event generators. It does this by means of a fortran77 common block, which specifies properties of the particular process being simulated such as the types of particles involved and their momenta. Colour flow in the diagrams requires particular attention and is encoded in the LHA. However, all of the Les Houches Accords attempt to hide such details and requirements from the user. Only tool developers have to concern themselves with them. More recently, the Les Houches Accord event record has been changed to a minimal XML-style structure, for clarity, simpler parsing and to side-step cross-language difficulties [5] and several parsers (in different languages) have been developed and are available. The accord has also been re-written to take into account potential new beyond the Standard Model physics models [6].

In this review, I shall briefly describe the publicly available, supported, documented codes which allow supersymmetric phenomenological calculations ${ }^{1}$. In each case, a link to a current web-site and a reference to the relevant manual is given. The default language of each program is fortran77, but if the code is written in a different language, it shall be detailed in this review at the point when the main functionality of the code is discussed. As time passes, it is foreseen that some of the links listed here will become out of date. The reader is advised to read the manual of any code they wish to use from the electronic arXiv web-site in order to find updated links to downloads etc. In addition, more accuracy and extended functionality will no doubt be added to the various programs as time passes. This

\footnotetext{
${ }^{1}$ In fact, a "quick guide" of SUSY tools was written over two years ago []]. The present review contains an updated and much more extensive overview of the field.
} 
guide is intended to serve as a snap-shot of documented, supported, publicly available SUSY phenomenological tools at the time of writing. It is not practical to continually up-date it as the state-of-the-art evolves. However, it should be mostly accurate for a good few years and there are plans to extend a Beyond the Standard Model tools repository [8] to a Wiki site, so that the authors of the codes may include up-dates to the accuracy/functionality as they occur. We shall not describe here any of the detector simulations. First, in section 2 , we shall describe codes that calculate MSSM SUSY spectra and decays. Then, in section 3 , we list matrix element generators, followed by event generators in section 4 . We then turn to constraints: in section 5 we review public SUSY dark matter codes, followed by other indirect constraint calculators in section 6. We review some of the algorithms required to perform global fits to SUSY models using indirect observables in section 7 . Finally, in section 8, we conclude and present a table summarising the functionality of the SUSY tools mentioned in this review.

\section{Spectrum and Decays}

There are four publicly available dedicated MSSM spectrum generating codes, displayed in Table 1. They all solve the MSSM renormalisation group equations (RGEs) to two-loop order, subject to two sets of boundary constraints. One set of boundary constraint is at the weak scale, and matches the MSSM parameters to current data on Standard Model particle masses and couplings. It also ensures that electroweak symmetry is broken successfully by adjusting the MSSM $\mu$ parameter. The other boundary condition is typically at a high energy scale, and involves setting the SUSY breaking parameters according to some theoretical model of SUSY breaking mediation. Universal mSUGRA, minimal anomaly mediation and minimal gauge mediation are supported by all of the codes. In addition, non-universal models such as those that can be invoked by the SLHA are supported. Each of the codes supports different additional possible SUSY breaking models. They each also support the SLHA aside from ISAJET [9]. An unofficial version of ISAJET which outputs in SLHA format does exist, however [10].

\begin{tabular}{c|cccc} 
Name & Language & RGEs & comment & manual \\
\hline ISAJET & & dominant 3rd & $\nu_{R}$ & 9 \\
SOFTSUSY & C++ & 3-family mixing & & 11 \\
SPheno & fortran90 & 3-family no-mixing & $\nu_{R}$ & 12 \\
SUSPECT & & dominant 3rd & & 13 \\
\hline
\end{tabular}

Table 1: SUSY Spectrum generators. $\nu_{R}$ indicates that the program includes an option for including right-handed neutrinos in the spectrum in order to obtain neutrino masses. Dominant 3rd RGEs mean that all Yukawa couplings other than the third family's are neglected in the RGEs whereas 3-family no-mixing means that all diagonal Yukawa couplings are included.

The details of approximations within the codes can be found within the manuals, and although similar, do tend to vary somewhat. They may differ by higher-order corrections, for example. The matching conditions to current data at the weak scale is mostly in the one-loop approximation. But when one is correcting a QCD cross section with 


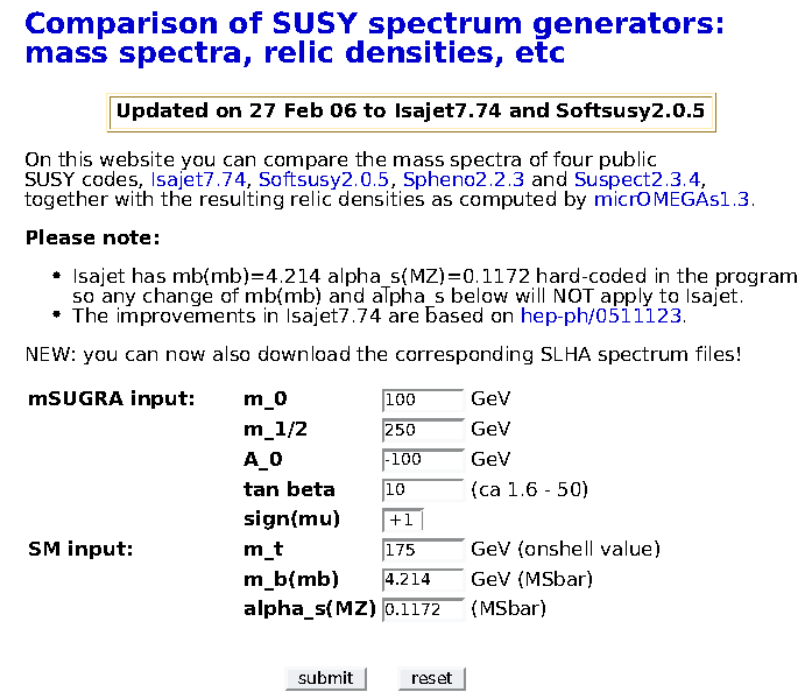

Figure 2: Comparison web-page mSUGRA form [14]

stop loops, for example, in order to extract the MSSM value of $\alpha_{s}\left(M_{Z}\right)$ that should be used, the question arises which stop mass should be used? The codes either use pole masses or running masses evaluated at different scales for the stops running in the loops. The difference between these choices is actually a higher order effect, and could only be fixed were the two-loop matching conditions known. Broadly speaking, RGE evolution is two-loop, in different approximations, as displayed in Table 1. ISAJET differs from the other codes in that it decouples sparticles at their mass scales, thus re-summing terms $\sim \mathcal{O}\left(1 /\left(16 \pi^{2}\right) \log [\Delta M / M]\right)$, where $\Delta M$ is the splitting between two sparticles and $M$ is their average mass. On the other hand, the other three codes all evolve using MSSM RGEs above $M_{Z}$ without decoupling sparticles, but then one-loop decoupling effects are added to the weak-scale boundary condition to leading logarithmic order. This latter approach allows the easy addition of some one-loop finite pieces, some of which are missed by the mass-scale decoupling approach taken by ISAJET. In summary, one may expect the massscale decoupling approach to provide a more accurate answer when sparticle splittings are very large, and $M_{Z}$ decoupling including all finite terms to be more accurate for a more typical sparticle splitting. The codes all agree to the percent level, except in particularly difficult parts of parameter space such as the focus point or very large $\tan \beta$ [15], where the differences can be much larger due to inherent numerical instabilities and the size of higher order corrections in those regions. Fortunately, a web-site exists [14] where one can input a SUSY breaking point on a web form as exemplified in Fig. 2, and quickly compare the output from the different codes. If one is doing a study on a particular point, for example, this provides a quick practical way of finding out if the point comes with particularly large theoretical uncertainties or not. 


\subsection{MSSM Sparticle Decays}

Currently, the programs that calculate sparticle decay branching ratios are Herwig++ 16 $(\mathrm{C}++)$, ISAJET [9], MadGraph [17], PYTHIA [18], SDECAY [19] and SPheno [12]. SDECAY, PYTHIA and Herwig++ take the SLHA stream from any of the other codes in order to produce SLHA-compliant output including decay information, whereas the other two codes are linked to their spectrum generators. The decay packages implement tree-level two-body decays of fermions and gauginos and three-body decays of charginos, neutralinos and gluinos. SPheno includes gluonic QCD corrections into decays by quarks. SDECAY implements some three and four-body decays of top squarks and one-loop corrections to the two-body decays. PYTHIA and Herwig++ contain internal routines for calculating sparticle decays, including tri-linear $R$-parity breaking effects. Herwig++ and MadGraph include angular correlations between subsequent decays in a sparticle cascade decay using the pioneering techniques of ref. [20], whereas all the other codes make a phase-space approximation. The program BRIDGE [21] was written in order to decay particles passed to it by matrix element generators in general models defined in the MadGraph format, then pass them on to showering and hadronisation programs. It calculates two and three-body tree-level decays itself, while keeping track of initial vertex spin structures via HELAS. Typically, phase-space is a reasonable approximation in hadronic collisions unless one is trying to fit the spin of sparticles.

\subsection{Higgs Masses and Decays}

There are some packages specialising in SUSY Higgs calculations: FEYNHIGGS 22] calculates the Higgs masses in a Feynman diagrammatic approach. In calculating Higgs masses, important two-loop effects are included for the MSSM with or without complex parameters, with a re-summation of the leading (s)bottom corrections. One-loop non-minimal flavour violating corrections to Higgs masses/mixings are included at the one-loop level. The program calculates the Higgs spectrum and decays and provides an estimate of theoretical uncertainties in the prediction. The two-body tree level decays include dominant one-loop corrections and the Higgs decays to $g g$ and $\gamma \gamma$ include all of the MSSM particles in the loop. A FEYNHIGGS web-form interface exists for checking single points in parameter space [22]. The program CPsuperH [23] also performs MSSM Higgs calculations when CP violating phases are present, including some effects up to two-loop order. The program is based on renormalisation-group improved diagrammatic calculations that include logarithmic as well as threshold corrections and $b$-quark Yukawa coupling re-summation. Some dominant one-loop pieces are included in the Higgs decays, which can be into SUSY or SM particles (including some important three body decays). The Higgs couplings and neutral Higgs mixings are also provided by the program. HDECAY [24] calculates up to three-loop QCD corrected decays of Higgs bosons in the CP-conserving MSSM where expressions exist in the literature, including some loop-induced decays, decays into two massive gauge bosons, three-body decays and decays into SUSY particles. Leading electroweak corrections are included (they can become important in the large Higgs mass regime due to enhanced Higgs self-interactions). All MSSM particles are included in the loop for the calculation of $\gamma \gamma$ and $g g$ Higgs decay modes. The leading QCD corrections are included for the gluonic mode. 
FCHDECAY 25] computes the flavour changing neutral current decays $B R\left(H^{0} \rightarrow \bar{t} c, t \bar{c}\right)$ and $B R\left(H^{0} \rightarrow \bar{b} s, b \bar{s}\right)$ in the flavour violating MSSM, using SLHA2 for input/output. It includes full one-loop SUSY QCD contributions.

\subsection{NMSSM}

The addition of a Standard Model singlet superfield to the MSSM constitutes a potential solution to the $\mu$ problem (why $\mu$ is of order the electroweak scale rather than some much heavier scale) and is called the next-to-MSSM (NMSSM). In the package NMSSMtools [26], sparticle masses are calculated using two-loop NMSSM RGEs in the dominant third family approximation. Tree level sparticle decay widths and branching ratios are also calculated. The Higgs masses, couplings and widths (for two-body modes) are calculated within the NMSSM using approximations to the one and two-loop dominant corrections. For decays into the SM particles, the widths are calculated including one-loop SM QCD corrections.

\section{Matrix Element Generators and Cross Section Calculators}

In the high-energy LHC régime, often we wish to calculate the production of more than two hard particles. This is the job of matrix element generators. Matrix element generators can usually calculate total or differential cross-sections and/or produce independently sampled events. Simulating (for example) the production of two squarks plus some additional hard QCD radiated jets requires us to deal with complicated Feynman diagrams involving many particles in the final state. For this job, one uses a matrix element generator, which simulates or calculates the hard process (e.g. $\left.q \bar{q} \rightarrow \tilde{t}_{1} \bar{t}_{1}+j e t\right)$. The matrix element generators are currently mostly at tree-level, particularly as regards SUSY physics. In practice, $2 \rightarrow 6$ to $2 \rightarrow 8$ processes may be feasible depending upon the number of Feynman diagrams, although a vast amount of CPU time may be needed to compute them (using, for example, the grid). The number of Feynman diagrams tends to grow to be too large with increasing numbers of final-state particles.

FeynArts/FormCalc [27] are Mathematica packages for the generation and calculation of Feynman diagrams up to one-loop order. They can thus be used to calculate matrix elements for scattering processes. Up to $2 \rightarrow 3$ processes can be calculated at the oneloop level with integration optimisation, although FormCalc has been successfully used to compute $2 \rightarrow 4$ processes at tree-level. Vanilla, CP violating and non-minimal flavour violating versions of the MSSM are available. There is also a way of encoding some new physics Lagrangian model for extensions. FormCalc simplifies the amplitudes generated

by FeynArts analytically and generates fortran77 code for the numerical evaluation of the squared matrix element. Automatic generation and pictorial representation of Feynman diagrams is also supported, as is convolution with parton density functions (PDFs). Recently, a program HadCalc 28] has been developed based on FeynArts and FormCalc. It takes the output from those codes in terms of partonic cross sections and convolutes them with PDFs. There are convenient ways to place cuts and an interactive menu-driven front-end that can be used to dial in SUSY parameters. 
Currently, CalcHEP [29] and CompHEP [30] (C) $)^{2}$ can cope with up to 6 external legs in a Feynman diagram, for example $(1 \rightarrow 5$ or $2 \rightarrow 4)$. The two programs can produce $\mathrm{C}$ output of analytical expressions for subsequent compilation and use. They have graphical interfaces which can display modulus squared Feynman diagrams. Models are already defined for the MSSM, NMSSM and the CP-violating MSSM. For the encoding of model Lagrangians and parameters, LanHEP [31] (C) is used. If the user wishes to extend some SUSY model outside of the ones already defined, LanHEP provides the means. MadGraph 17 performs vanilla MSSM matrix element calculation with SLHA input. Helicity amplitudes are constructed based on the HELAS [32] library in order to encode spin information of the produced particles, which can be used in their decay. Feynman diagrams are drawn, and fortran77 output is produced for the matrix element. A Monte-Carlo integrator package has been included in MadGraph, and SUSY differential or total cross sections can be calculated using it. Alternatively, the final result can be Les Houches Accord formatted parton-level events that can be passed into an event generator for subsequent parton showering and hadronisation. The MadGraph web-site has a form that can be filled in to get events returned automatically. The event generator SHERPA [33] utilises an event generator Amegic++ 34] (C++) that also uses the helicity amplitude technique and calculates at the tree-level, with the possibility of up to six particles in the final state of the hard scattering. Whizard [35] (fortran95) includes initial and final state polarisations and can calculate in the vanilla MSSM as well as the CP-violating case. It uses O'Mega [36] (0' Caml) to translate a helicity amplitude into computer code as needed. O'Mega is designed with special tricks to avoid the factorial increase in CPU time with the number of external particles. It has been demonstrated to work for some processes with eight particles in the final state. SUSYGEN [37] is restricted to $2 \rightarrow 2$ SUSY production processes. It can include polarisation in $e^{+} e^{-}$collisions and covers vanilla as well as R-parity or CP-violating MSSM models. GRACE [38] performs computations of $e^{+} e^{-} \rightarrow$ up to four bodies in the MSSM at tree-level. GRACE draws the relevant Feynman diagrams for the user.

Numerical results of several hundred SUSY production cross-sections were compared between MadGraph [17], SHERPA [33] and Whizard [35] and they were all found (eventually) to agree [39]. PROSPINO 40 (fortran90) computes MSSM next-to-leading order cross sections for the production of two sparticles at hadron colliders. It can also cope with the production of weak gauginos in the split SUSY framework. Detailed calculations of crosssections of $e^{+} e^{-} \rightarrow$ sleptons at the one-loop level are also available from ILCslepton [41]. FeynHiggs [22] calculates Higgs production cross-sections for the Tevatron and the LHC including SUSY corrections at the production vertex.

\section{Event Generators}

The most well-known general purpose SUSY event generators (PYTHIA [18], Herwig++ [16]) usually implement a hard-sub process in terms of two particles scattering on two particles, represented by the central vertex in Fig. 3. The initial particles in this hard-sub process

\footnotetext{
${ }^{2}$ These two programs have the same origin, but at some stage the development of them branched. Because of this, although the programs are now different, many features of them are similar.
} 


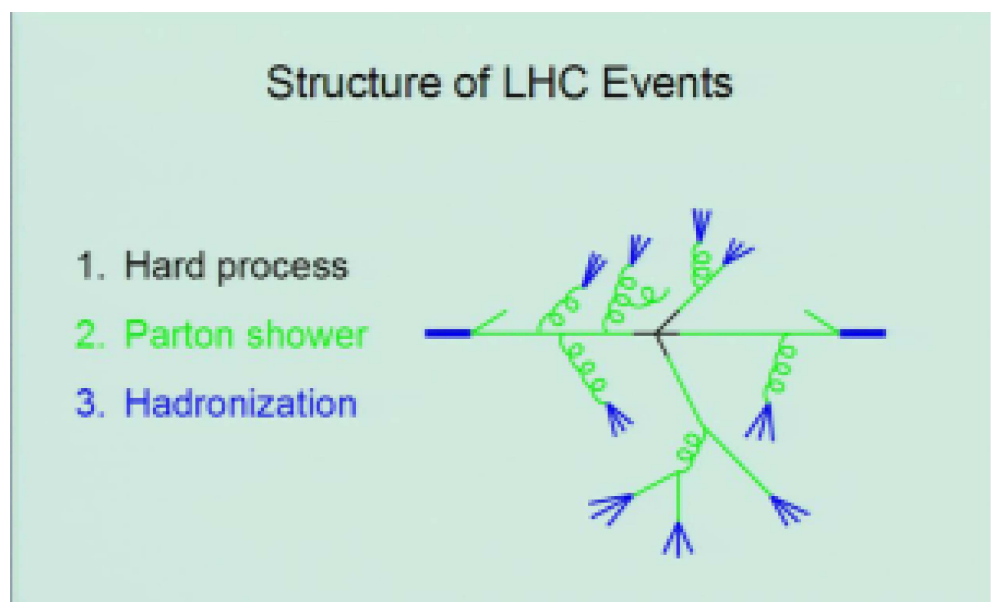

Figure 3: Schematic of a hadron collision simulation in an event generator.

may be leptons, or point-like constituents of hadrons. In the latter case, the quarks and gluons are extracted from a hadron by means of the parton density functions. The hardsub process is usually calculated at leading order in perturbation theory within event generators, especially for exotic signals such as SUSY. If SUSY particles are produced in the simulated event, they are then decayed randomly, according to the branching ratios calculated by a program in section 2.1. The resulting cascade decay spits out SM particles, some of which may be quarks or gluons. These quarks and gluons then emit soft QCD radiation, which is modelled by the parton shower. Parton showering encodes the fact that the matrix elements of massless coloured particles emitting a gluon have a singularity in the infra-red or collinear limit. The initial state may also shower, emitting QCD radiation. It can be important to include effects in the shower coming from colour coherence in order to describe the resulting jets adequately. Various properties such as angular ordering of the shower (with preceding emissions being at smaller angles) are evident in the resulting event. Once the partons are showered down to some energy scale to be decided by the event generator, some non-perturbative modelling (and a tune to data) collects the partons together into hadrons which, after their decays have been simulated, may be observed in the detector. Finally, the simulated events are often represented by a series of lines in a text file (representing variables held in memory), each line describing the kinematics and state of a particle involved with the event. Information on which particles decayed into which other particles is also indicated in this event record. We briefly mention some of the Standard Model properties of event generators, since elements of them are also relevant to SUSY events, but the interested reader should see ref. [42] for a more complete guide to Standard Model event generators in hadron collisions.

A general framework for encoding new physics models is included in Herwig++ [16] so that users may define the relevant particles and Feynman rules for the hard sub-process. The MSSM has already been defined within the framework, but extended SUSY models must be input by using it. It can read SLHA files for input SUSY information. The Herwig++ shower algorithm treats QCD radiation from coloured heavy objects (for example tops). 
It can evolve to zero transverse momentum of emissions, giving an improved simulation of the dead-cone effect for radiation from massive particles. An eikonal multiple scattering model is used for simulating additional partonic collisions in the same hadronic collision. Such processes form part of the underlying event. Herwig++ uses a cluster model for the hadronisation step, clustering quarks and gluons that have similar kinematics into colour singlet states, which decay to hadrons and hadron resonances. Herwig ++ treats its decays including spin correlations all of the way down the various decay chains. Herwig++ relies on an underlying C++ structure developed for high energy collisions called ThePEG 443].

PYTHIA [18], on the other hand, does not depend upon ThePEG, but is stand-alone. As well as hadron-hadron collisions, it can deal with $e^{+} e^{-}$beams. Initial and final-state parton showers are based on $p_{T^{-}}$-ordered evolution, terminating at $1 \mathrm{GeV}$. Although there is currently a C++ version in development, it does not contain any SUSY physics and so we concentrate on the older fortran77 version in this review. Many different options for the changing the models of parts of the PYTHIA simulation are possible, but here we describe the default models. Hadronisation and hadron fragmentation (decay) are modelled by the Lund string model, where hadrons are modelled to be a colour flux tube, ended where the (di-)quarks are located. The MSSM, the NMSSM, tri-linear $R$-parity violation, as well as long-lived coloured sparticles such as those that exist in models of split SUSY, are included in the PYTHIA distribution. Polarisation is included for $e^{+} e^{-}$incoming beams. All decays of sparticles are using the phase-space approximation, and so sparticle spin is not simulated.

A new event generator has recently been developed called SHERPA [33] (C++). The main design feature of SHERPA is that it combines parton shower evolution and matrix element generation. The MSSM with or without CP violation and full inter-generational mixing is included, as well as a general formalism (compatible with the matrix element generator Amegic++ [34], which ships with the SHERPA distribution) provided for adding new particles and interactions. Amegic++ can practically handle up to six jets in the final state for $e^{+} e^{-}$collisions, and up to three jets for hadron collisions. One of the difficulties of combining parton showers and matrix element generation for hard jets is the problem of double-counting. If one simply adds the matrix element generation for 3 jets to the 2 -jet plus parton shower sample, one could easily double count the region where one of the jets is soft (and therefore already included in the parton shower). In SHERPA, the parton shower evolution and matrix element generation are matched via the CKKW formalism [44], where the matrix element configurations are re-weighted according to a pseudo shower history and shower emissions that overlap with higher order matrix elements are rejected. SHERPA also performs the hadronisation/fragmentation step using its own cluster model [45], which includes di-quark spin effects and a dynamic separation of the régimes of clusters and hadrons according to their masses and flavours. When calculating SUSY decays in SHERPA, there is currently no facility for picking the decay products automatically, the user must supply which decay chain is required. After this has been done though, the spin information and off-shell effects are included in each sparticles' decay into the next sparticle and Standard Model particle.

The ISAJET [9] event generator simulates $p p, p \bar{p}$ and $e^{+} e^{-}$collisions at high energy, 
based on perturbative QCD and phenomenological models for parton and beam jet fragmentation, not including colour coherence effects: the probability of emitting a soft gluon is multiplied by a factor given by the Alterelli-Parisi function. ISAJET keeps only the parts that are in the exact collinear limit, but uses non-collinear kinematics. QCD radiation from initial and final states is simulated. Sparticle pair production at tree-level is supported, along with subsequent decay. The ISAJET hadronisation model is the independent fragmentation ansatz of Field and Feynman, which forms new (di-)quark-anti-quark pairs out of partons, and groups them together into mesons and baryons with some fraction of their summed momenta.

\section{Predictions of SUSY Dark Matter}

The recent WMAP5 cosmological fits to the cosmic microwave background and other data provide us with an accurate observation of the density of dark matter in the universe as a fraction of the relic density: $\Omega h^{2}=0.1143 \pm 0034$ [46]. The MSSM offers several possible candidates for weakly interacting massive particles that could play the rôle of cold dark matter, since the lightest supersymmetric particle (LSP) is stable from the assumption of $R$-parity. The dark matter candidate obviously must be without electric charge, so that it does not interact with light, and also should be colourless, otherwise it would have fused with nuclei during nucleosynthesis and been discovered in anomalously heavy isotope searches. Gravitinos and lightest neutralinos are possible candidates within the MSSM, although in extended models, other SUSY particles are possible dark matter candidates. In principle, SUSY dark matter may be discovered in direct detection experiments, where nuclear recoils from collisions with SUSY dark matter are possible. If the dark matter candidate interacts too weakly (for example in the case of the gravitino), the direct detection cross-sections are far too small to small to be seen in the foreseeable future. However, in the case of the neutralino, there is a chance for direct dark matter observation. In order to detect dark matter on earth, it is of course a necessary condition that there is some dark matter going through the detector. While only a small amount is empirically known about the small-scale structure of dark matter halos, numerical $N$-body simulations indicate that even if one starts with strict filaments or cusps of dark matter, subsequent Newtonian evolution will tend to smear it out. Thus, the prospect of having our galaxy's dark matter localised completely elsewhere in the galaxy seems unlikely, but it should be borne in mind that any calculation of the local dark matter flux on earth is subject to large astrophysical modelling uncertainties. Aside from the direct detection of particulate dark matter, there are prospects for indirect detection, where for example, dark matter annihilation in the sun, in the earth or in the centre of the galaxy produces high energy particles that can be detected on earth or on satellites.

Once a SUSY model's parameters has been fixed and a cosmological model is assumed, it is possible to estimate the amount of current dark matter relic density in the universe is predicted. The codes tend to assume the $\Lambda$ CDM model of a cosmological constant plus cold dark matter component, since this is quite a simple and good fit to the WMAP and large scale structure data. One has to track the abundances of the different species of 
sparticle through the early evolution of the universe. They can annihilate with each other into Standard Model particles, but eventually, the expansion of the universe makes the sparticles too far apart to interact. Aside from losses due to annihilation processes, each sparticle will end up as a LSP through its decays. The tracking of the abundances of the various sparticle species and involves the evolution of coupled Boltzmann equations. There are many different annihilation cross-section processes to consider, and the relevant public codes currently calculate at tree-level. The velocity distributions of the SUSY particles are derived from Maxwell-Boltzmann approximations. This can still involve the calculation of thousands of Feynman diagrams, however. As a consequence, the current tools calculate mainly at tree-level. However, loop corrections can give large $\sim O(10) \%$ effects in some cases 47.

DarkSUSY [48] contains hard-coded matrix elements for the many different annihilation processes of the vanilla MSSM. It can calculate the relic density as well as direct and indirect detection rates, with a choice of different nuclear form factors for the direct rates. Solar system WIMP velocity distributions can be used to calculate the capture in the Earth of dark matter particles. An exotic component of positron, anti-proton and antideuteron in cosmic rays originating from neutralino pair annihilation in the galactic halo can be calculated. Hadronisation and fragmentation was calculated with PYTHIA and the results tabulated from various neutralino masses, which DarkSUSY interpolates in order to provide an estimate of the particle yield. For particle yields coming from annihilation in the earth and the sun, 6 fundamental channels are included: $c \bar{c}, t \bar{t}, b \bar{b}, \tau^{+} \tau^{-}, W^{+} W^{-}$and $Z^{0} Z^{0}$. Recent solar and terrestrial density models are included as a necessary ingredient in the calculation. For galactic halo annihilations, $W^{+} W^{-}, Z^{0} Z^{0}, W^{+} H^{-}, Z^{0} h^{0}, Z^{0} H^{0}, h^{0} A^{0}$ and $H^{0} A^{0}$ channels are included, with subsequent decay of these particles, including the heavy quarks $c, b$ and $t$. The $g g, \gamma \gamma$ and $Z \gamma$ channels occurring at the one loop level are also included. Anti-matter production yields from dark matter annihilation in the Galactic halo are determined by DarkSUSY. Modelling the propagation of anti-matter is non-trivial, but DarkSUSY attempts this through various approximations which can be found in the manual. High energy neutrinos and neutrino-induced muons can be detected by neutrino telescopes and their yields are calculated. The SLHA is currently not supported, but instead dedicated interfaces to ISAJET and SUSPECT are included for spectrum generation. There is a web interface linked from the DarkSUSY homepage for inputting a MSSM model and calculating the relic density and detection cross-sections. Thus, if one is doing an analysis on one point in parameter space, one can check its dark matter properties easily on-line.

IsaRED is part of the ISAJET [9] package, and calculates the relic density of neutralino dark matter in the MSSM. Annihilations between $\chi_{1}^{0}, \chi_{2}^{0}, \chi_{1}^{ \pm}, \tilde{e}_{1}, \tilde{\mu}_{1}, \tilde{\tau}_{1}, \tilde{\nu}_{e}, \tilde{\nu}_{\mu}, \tilde{\nu}_{\tau}, \tilde{u}_{1}, \tilde{c}_{1}$, $\tilde{t}_{1}, \tilde{d}_{1}, \tilde{s}_{1}, \tilde{b}_{1}$ and gluinos are taken into account in the calculation. In the same package, IsaRES evaluates spin-independent and spin-dependent direct detection rates. Squark, $Z^{0}$ and Higgs exchanges are included at tree-level and neutralino-gluon interactions involving quarks, squarks and Higgs bosons are included at the one-loop level.

micrOMEGAs (C) 49] calculates the relic density of the LSP at tree-level and direct/indirect detection rates in the vanilla MSSM, the MSSM with complex phases and the NMSSM. 
Important higher-order QCD and SUSY QCD corrections to Higgs quark vertices are included. The program can be used to calculate the relic density of a charged and/or coloured next-to-lightest supersymmetric particle. This can be useful in the case of a gravitino LSP. Gravitinos are not simulated by micrOMEGAs, but a simple formula can be used to extract their relic density from the relic density of the next-to-lightest supersymmetric particle. The most important annihilation channels for any given model point can be output. micrOMEGAs uses CalcHEP [29] in order to calculate any necessary Feynman diagrams, and so extensions can be encoded using the LanHEP [31] Lagrangian formulation, for models where there is only one stable particle. Only diagrams that may contribute up to some specified fraction (by default, $10^{-5}$ ) of the thermally averaged total annihilation cross-section are included, which makes for faster computation. LSP scattering rates on nucleons and nuclei in the spin-independent and spin-dependent interaction cases are also presented. $\gamma, e^{+}, \bar{p}$ and $\nu$ yields for indirect direction purposes (at $v \rightarrow 0$ and/or in the continuum) are calculated. Like DarkSUSY, micrOMEGAs uses the basic channels $q \bar{q}, \mu^{+} \mu^{-}, \tau^{+} \tau^{-}, W^{+} W^{-}$and $Z^{0} Z^{0}$ and interpolates tables of $\gamma, e^{+}, \bar{p}$ and $\nu$ production as obtained by PYTHIA. For channels that contain two different particle species $A B$, the final spectrum is obtained by taking the average of $\mathrm{di}-A$ production and $\mathrm{di}-B$ production as a rough approximation. The galactic gamma-ray flux is calculated with a modified isothermal distribution of dark matter in the galaxy. For direct detection rates, higher order corrections to Higgs-quark vertices and one-loop neutralino-gluon interactions are included for the vanilla MSSM, the CP-violating MSSM and the NMSSM.

\section{Predictions for constraints}

There are many constraints upon supersymmetric models: direct constraints tend to be the easiest to implement, being (usually) phrased as lower bounds on sparticle masses. Relevant indirect constraints are upon branching ratios for rare decays, precision electroweak observables or electric dipole moments for example, and often occur at the loop level. Being of general utility, FormCalc [27] can be used to calculate the relevant SUSY matrix elements.

\section{1 $b$ observables}

The branching ratio of $b \rightarrow s \gamma$ has long been used to constrain supersymmetric models, and is calculated by several codes. Many of the codes calculate it in the vanilla MSSM without SUSY flavour mixing. For minimal flavour violating MSSM computations, SusyBSG [50] calculates the branching ratio for the decay $b \rightarrow s \gamma$ taking into account all of the available next-to-leading order (NLO) contributions, including the complete supersymmetric two-loop QCD corrections to the Wilson coefficients of the magnetic and chromo-magnetic operators, as well as an improved NLO determination of the relation between the Wilson coefficients and the branching ratio. micrOMEGAs [49], predicts the branching ratio including next-to-leading order contributions for the Standard Model. The charged Higgs and supersymmetric large $\tan \beta$ effects beyond leading-order are included. DarkSUSY 48 performs a NLO calculation which is complete for the Standard Model prediction and 
adds some dominant NLO MSSM corrections. SPheno [12] and SUSPECT [13] include oneloop MSSM corrections and some NLO corrections to the branching ratio. SuperIso [51 calculates the $b \rightarrow s \gamma$ branching ratio in the vanilla MSSM with flavor violation, NLO supersymmetric contributions and next-to-next-to-leading order (NNLO) Standard Model contributions. Flavour violation is supported through the SLHA2 interface. SuperIso is currently the only code to predict the isospin symmetry breaking $\Delta_{0-}$ of the $B \rightarrow K^{*} \gamma$ decay including the NLO SUSY contributions. CPsuperH [23] can provide a prediction for the branching ratio as well as as its CP-asymmetry and SUSY contributions to $B_{s, d}^{0}-\bar{B}_{s, d}^{0}$ mass differences $\left(\Delta M_{s, d}\right)$. FeynHiggs [22] provides a prediction for the $b \rightarrow s \gamma$ branching ratio including non-minimal flavour violating effects. Another $b$-physics observable that can constrain SUSY is the to-date unobserved rare decay mode $B_{s} \rightarrow \mu^{+} \mu^{-}$. The SUSY calculation in micrOMEGAS [49] includes the one-loop contributions due to chargino, sneutrino, stop and Higgs exchange. $m_{b}$ re-summation effects at high $\tan \beta$ are taken into account. CPsuperH [23] also performs the calculation of $B R\left(B_{s} \rightarrow \mu^{+} \mu^{-}\right)$in the CP violating MSSM, as well as $B_{d} \rightarrow \tau^{+} \tau^{-}, B_{u} \rightarrow \tau^{+} \nu_{\tau}$. Each branching ratios is calculated in the single-Higgs insertion approximation. NMSSMtools [26] calculates $b \rightarrow s \gamma, B_{s} \rightarrow \mu^{+} \mu^{-}$, and $B^{+} \rightarrow \tau^{+} \nu_{\tau}$ branching ratios as well as $\Delta M_{s, d}$ in the NMSSM at one-loop order. ISATOOLS [9] includes NLO contributions to some of the Standard Model Wilson coefficients for $B R(b \rightarrow s \gamma)$ and one-loop MSSM corrections. Branching ratios for $B_{s} \rightarrow \mu^{+} \mu^{-}$ and $B_{d} \rightarrow \tau^{+} \tau^{-}$are calculated to one-loop, using approximations for the chargino masses (neglecting their mixing). The fitting program SuperBayes [52] uses the micrOMEGAs prediction of $B R(b \rightarrow s \gamma)$ at NLO and then augments it by NNLO Standard Model QCD contributions.

\subsection{Anomalous magnetic moment of the muon}

The anomalous magnetic moment of the muon is currently around $3 \sigma$ higher than the Standard Model prediction. There is thus room for a non-zero SUSY contribution. ISATOOLS 9], SPheno [12], SuperIso [51], micrOMEGAS [49] and DarkSUSY [48] calculate the predicted SUSY contribution to one-loop order, whereas FEYNHIGGS [22 and SUSPECT [13] also include some two-loop corrections.

\subsection{Electric dipole moments}

For calculations of electric dipole moments in the CP-violating MSSM, micrOMEGAS 49 can provide estimates for the electron and Thalluim. One-loop neutralino/chargino contributions and two-loop squark, quark and chargino contributions are included as well as four-fermion operators for Thallium. Two-loop Higgs-mediated contributions to electron, muon and Thallium electric dipole moments are calculated in CPsuperH 23]. However, currently some well-known one-loop contributions have yet to be implemented. The Thallium, neutron and mercury electric dipole moments are calculated in FEYNHIGGS 22].

\subsection{Electroweak observables}

micrOMEGAs [49] and SUSPECT [13] can output the $\Delta \rho$ parameter, which describes some loop corrections to electroweak observables. They both contain one-loop stop/sbottom 
contributions, as well as two-loop QCD corrections due to gluon exchange and the heavygluino limit of gluino exchange. FeynHiggs [22] also contains a calculation of $\Delta \rho$, with corrections up to two-loops. SPheno [12] outputs the one-loop sfermion contributions to $\Delta \rho$. In terms of the electroweak observables themselves, FeynHiggs also computes $M_{W}$ and $\sin ^{2} \theta_{w}^{e f f}$ including some two-loop SUSY contributions, non-minimal flavour violating effects and the effect of complex phases in the stop/sbottom sector at one-loop.

\section{Fitting tools}

We first introduce some necessary statistical terms, then go on to discuss their use in the context of SUSY fits. Typically, global fits of models to data utilise a statistical "figure of merit" for each point in parameter space to characterise how well it fits data. The most familiar one for particle physicists is probably $\chi^{2}$, but sometimes likelihood is used instead. Likelihood $L$ can be simply related to the $\chi^{2}$ parameter, $L \propto e^{-\chi^{2} / 2}$. $L$ or $\chi^{2}$ are often quoted in frequentist statistical interpretations of data. Bayesian statistics turn these quantities into probability distributions on the input parameters of the model, requiring the introduction of the infamous prior probability distribution. The probability distribution of some parameter after confrontation with data is called the posterior probability distribution. A global fit of some model to data often consists of finding the variation of the figure of merit with the model parameters. The best-fit set of model parameters is sometimes quoted, with the amount of parameter space contained within some expected amount of statistical variation of data. More complete analyses map out the figure of merit on the parameter space, and Bayesian analyses then make probabilistic inferences based upon the map.

\subsection{Algorithms for multi-dimensional fits}

Even if one restricts the MSSM to some lower number-of-parameters form such as mSUGRA, the parameter space is still of considerable dimensionality: 4 for a given sign of $\mu\left(m_{0}, M_{1 / 2}, A_{0}\right.$ and $\tan \beta)$. Also, if one wants to perform global fits of the model to data, one should include variations of the relevant Standard Model input parameters. $m_{t}$ is proportional to the largest parameter in the model, the top Yukawa coupling, and for high $\tan \beta$ the bottom Yukawa coupling, proportional to the bottom quark mass, can change the predicted values of observables. Variations of $\alpha_{s}$ within its empirical uncertainties can also have a large effect on squark and gluino masses through the RGE evolution, since it is the largest gauge coupling. If one is including precision electroweak observables in the fit, including uncertainties on the fine structure constant $\alpha$ becomes essential. Thus, in mSUGRA one has an eight-dimensional relevant parameter space. Scans in such a space are impractical, since the required number of points is exponential in the number of parameters. If one required a resolution of 25 points for each parameter, $1.5 \times 10^{11}$ points would be required in total. To make matters worse, there are often sharp features in the $\chi^{2}$ distribution that would render such a low resolution insufficient. Such a large number of points cannot be calculated in a reasonable amount of CPU time, even given recent advances in computer technology. If one has access to a computer farm, calculating a few million points is feasible 
within a few days, for example (unless one wants to simulate event generation, which would take much longer). There is therefore a need for more sophisticated scanning algorithms that can reduce the required number of scanned points for parameter spaces of more than three dimensions.

The software tool MINUIT [53] is a well-tried function minimiser. It calculates derivatives of the figure of merit with respect to input parameters and performs hill-climbing algorithms to try to find the best-fit point. It then determines the error matrix from a matrix of second derivatives of $\chi^{2}$. This error matrix contains information about the $1 \sigma$ standard deviations of the parameters in the Gaussian approximation (where $a \chi^{2}$ is assumed to be parabolic around the best-fit point) and including correlations. For cases where the Gaussian approximation is a bad approximation, another internal MINUIT algorithm can be used for determining errors including non-linearities, but can be very time consuming depending upon the amount of non-linearity. Algorithms that use derivatives can be problematic when the surface that they are minimising are rough. In the SUSY fitting case, the original SUSY spectrum is obtained by an iterative process up to some numerical accuracy, which then feeds into the rest of the figure of merit calculation, providing small discontinuities in the surface. A typical numerical fractional accuracy in this stage of the calculation might be $10^{-3}$. While a fractional accuracy of $10^{-5}$ is feasible, it requires much more CPU time per scanned point, and is actually unattainable in certain "difficult" regions of parameter space such as the focus-point region. MINUIT also finds parameter degeneracies problematic, where the figure of merit does not change much along some curve in parameter space. Despite these short-comings, MINUIT has been used to perform global fits of mSUGRA to global data successfully [54].

MCMC methods are commonly used in cosmological [55, 56] and other contexts, and recently there has been a realisation that they are very useful to the SUSY high-dimensional scanning problem. MCMCs scan more often where the fit is good and the figure of merit is high and less often in the tails of distributions. In fact, the density of scanning is proportional to the figure of merit. MCMC methods have a high CPU overhead, meaning that they are not the most efficient tool for one or two dimensional problems. But the required number of points goes roughly linearly with the number of dimensions rather than exponentially, and so they are very useful for our higher-dimensional mSUGRA fitting problem. In this context, a Markov chain consists of a long list, or "chain" of points and their associated likelihoods. Statistical inference can be made by binning these points in terms of some quantity of interest. The simplest implementation of MCMC is called the Metropolis algorithm [57]. In the Metropolis algorithm, for the first point in the chain, a point $x_{0}$ is picked at random in parameter space and its posterior density calculated, $p_{0}$. A potential next point $x_{1}$ is picked in the vicinity of the previous point, again at random. If $p_{1}>p_{0}$, the new point is accepted. Otherwise, the new point is accepted with probability $p_{1} / p_{0}$. If the new point is not accepted, the previous point is added again on to the chain. This algorithm is repeated many times, until it has explored all of the relevant parameter space. There are many choices of how to pick a potential next point "in the vicinity" of the current one, and some trial and error is usually involved in setting the length scales involved. Usually, a Gaussian function is used to randomly choose the distance of the new point away 
from the current one, but formally, any well behaved function would work in the limit of an infinite number of MCMC steps provided it has no true zeroes. For efficient scanning, the length scale should be of order the length scale of the likelihood variation. If it is much larger, hardly any new points will be accepted and the efficiency will be too low. If it is much smaller, many new points will be required to explore all of the good-fit parameter space. In order to verify that the algorithm has indeed explored the parameter space properly, it is good practice to run several statistically independent chains concurrently. One can then compare the results in the different chains statistically to see how similar they are [58]. The Metropolis algorithm does not rely on derivatives and is therefore immune to serious problems caused by roughness from numerical error. It can easily be used to interpret data in a Bayesian form or in a frequentist form. For the Bayesian inference, one plots the quantities in question (say, squark mass vs gluino mass) in bins. The marginal posterior probability distribution in terms of these parameters is then proportional to the number of points in the chain that land in each bin. Marginal refers to the fact that all other parameters have been integrated over. In order to interpret the chain in a frequentist fashion, one plots the profile likelihood: the likelihood of the maximum likelihood point that lands in each bin [59]. Such a procedure, provided a sufficient number of samples to get near the maximum for each bin has been obtained, is equivalent to minimising $\chi^{2}$ in each bin. Confidence limits can be found in the parameter plane in question by plotting iso- $\Delta \chi^{2}$ contours, where $\Delta \chi^{2}$ is $\chi^{2}$ assigned to each bin minus the $\chi^{2}$ of the minimum bin. MCMC methods thus provide full maps of the figure of merit across parameter space or other scalar quantities that one is interested in. A package SuperBayes [52] (fortran77, fortran90 and C++) is available for performing global fits to SUSY models using MCMC and SOFTSUSY [11], DarkSUSY [48] and FEYNHIGGS [22]. The MCMC routines were adapted from cosmomc [56], as well as some of the plotting routines. The program SFITTER [60] is currently being developed which will fit SUSY models to collider data on sparticle masses using MCMC methods.

A problem that is not addressed by either MINUIT or by the Metropolis algorithm is that of well-separated $\chi^{2}$ minima. MINUIT only finds a local minima. In principle, the Metropolis algorithm may find all local $\chi^{2}$ minima in the limit of infinite number of samples. In practice however, if the local minima are small and require small length scales for suggesting proposed points, and the distance in parameter space between them is large, the chance to "hop" from one local minimum to the other may be tiny and require an unfeasibly large number of samples. A "tweak" to the Metropolis algorithm exists which can solve this problem and is called bank sampling [61]. In bank sampling, one performs a two-step process. In the first step, many different Metropolis chains are started and run for a small number of steps, but numerous enough to find points somewhere near local likelihood maxima. These points then form the "bank" or "cache" of points used in a new modified Metropolis algorithm. On each MCMC step, there is a small probability that the chain will propose a point in the vicinity of one of the bank points. If the new point is added successfully to the chain, the chain "teleports" to the other local maxima. In this way, the relevant local maxima all appear in the fit results, correctly normalised with respect to each other. 
If only the global likelihood maximum, or equivalently, the global $\chi^{2}$ minimum, is desired, a different modified Metropolis algorithm called simulated annealing can be used. Simulated annealed can be used to find a point near a global $\chi^{2}$ minimum when several local ones exist. In simulated annealing, it is imagined that the $\chi^{2}$ surface is some potential energy surface upon which a particle moves. A finite temperature is set, which increases the length scale of the proposal step (or, in the analogy, the average distance the particle moves). The temperature is very large at the start of the algorithm and gradually decreases to one thereafter. The chance of acceptance of a worse-fit point is also fixed to be higher with increased temperature $T$, being set to $e^{-\Delta \chi^{2} / T}$, where $\Delta \chi^{2}$ is the $\chi^{2}$ difference between the current point and the proposed worst-fit one. In the early stages, the algorithm is more likely to traverse bad-fit regions and not be trapped in local minima. The computer code FITTINO [62] can fit a 24-parameter simplified weak-scale MSSM to assumed cross-section and mass from SUSY signal collider data. Tree-level values of observables and subsets of SUSY parameters are used to obtain start values for the $\chi^{2}$-fit. Simulated annealing is then performed in order to find a better approximation to the global $\chi^{2}$ minimum. Using these parameter values, MINUIT is performed in order to minimise $\chi^{2}$ more precisely. In order to investigate the uncertainties in the fit, a series of fits for many imagined experimental data are performed in FITTINO, with data smeared around their nominal values, and the global $\chi^{2}$ minimum is found in each case.

In frequentist statistics, hypothesis testing often reduces to finding the minimum $\chi^{2}$ of different models. However, in Bayesian statistics, one wishes to calculate the evidence ratio: the ratio of volumes under the posterior probability surfaces, a quantity that can be very computationally intensive to calculate. Bank sampling provides a method for the rough computation of the evidence ratio, by having bank points within each of the separate models. After the MCMC has run, the ratio of points in each model is an estimate of the evidence ratio. Such an estimate may not be very accurate, particularly where the evidence ratio is much larger or smaller than one. In such cases, one can artificially multiply one of the model's likelihoods by a factor which will bring the resulting evidence ratio closer to one. The normalisation can be un-done, with the result that the ratio can be computed with smaller statistical uncertainty from the likelihood re-scaling. The disadvantage of bank sampling for Bayesian evidence evaluation is that only ratios of the evidence can be determined, not the evidence value on its own.

An algorithm which solves this problem as well as the well-separated likelihood maxima problem in a completely different way is the 'MultiNest' technique [63]. MultiNest models the multi-dimensional likelihood surface with a series of (possibly overlapping) ellipsoids. Clustering algorithms are contained within the larger algorithm. They determine when an ellipsoid is to be broken up into two different ellipsoids because the initial one does not model the underlying distribution well enough. Many live points are chosen, sampled from the prior probability distribution. Current live points are described in terms of ellipsoids, determined by the covariance matrix of the live points, enlarged by about $20 \%$ to take nonlinearities into account. The live point with the smallest likelihood is replaced with one with a higher likelihood re-sampled from the ellipsoids. Thus, the live points gradually home in on the likelihood maxima as the algorithm proceeds and the evidence can be calculated 
from the list of live points and their evidences, as can posterior probability inferences. The evidence of a single model can be accurately calculated in this approach, in contrast to the case of bank sampling. Thus, as one builds up a list of different models that one is testing against some set of data, there is no need to run many different comparisons between the different pairs of models: a single computation for each model suffices. The nested approach does need to be able to sample efficiently from the prior probability distribution and so will not work efficiently in cases where there is no analytic form for the prior. For extremely high dimensional cases (say, 10 and above), a MCMC-hybrid nested sampling approach may be more efficient than the ellipsoidal approach [63] for multi-modal distributions.

\subsection{Example Global Fits to mSUGRA}

We now display some example results using the various techniques introduced in section 7.1 . We pick examples of global fits to mSUGRA in the literature as an example. Typically, the data that authors have chosen to fit to include the relic density of dark matter to the relic density of neutralinos, $M_{W}, \sin ^{2} \theta_{w}^{e f f}, B R(b \rightarrow s \gamma), B R\left(B_{s} \rightarrow \mu^{+} \mu^{-}\right),(g-2)_{\mu} m_{t}, m_{b}\left(m_{b}\right)$, $\alpha_{s}\left(M_{Z}\right), \alpha\left(M_{Z}\right)$ and direct exclusion limits from colliders. Fig. 位a shows the posterior probability distribution in terms of the $m_{0}-M_{1 / 2}$ plane for both signs of $\mu$ with flat priors in $m_{0}, M_{1 / 2}, \tan \beta$ and $A_{0}$ after an MCMC fit using bank sampling [59]. The probability relative to the one in the maximum-posterior bin is shown by the colour, and measured by the bar on the right. The most probable region at low values of $m_{0}$ and $M_{1 / 2}$ corresponds to the stau co-annihilation region, where the lightest stau and lightest neutralino are quasimass degenerate. The extended probability mass at $m_{0}, M_{1 / 2} \sim 0.5 \mathrm{TeV}$ corresponds to the $A^{0}$ boson resonance at high values of $\tan \beta$, where dark matter annihilation proceeds efficiently through $\chi_{1}^{0} \chi_{1}^{0} \rightarrow A^{0} \rightarrow b \bar{b}$. At larger values of $m_{0}$, we have the focus point region, where efficient annihilation into weak gauge boson pairs is possible. In Fig. đtb, the same data is interpreted in a frequentist fashion using the profile likelihood technique. This technique picks out the best-fit points, rather than averaging over all points in the unseen dimensions. Figs. Aa, b differ where there are significant volume effects, that is where the volume of points in the unseen dimensions enhances or diminishes the Bayesian fit. The fact that the frequentist interpretation differs from the Bayesian one can be seen as a signal that more data is required for the fit; indeed we should not be surprised since a complex model with eight free parameters has been fit with some fairly indirect data. Similar fits to the Bayesian ones above were performed using the Metropolis MCMC algorithm, resulting in quite similar posterior probability densities for the particle physics properties, despite some differences in the indirect constraints used [64]. In addition, ref. [64] constrains dark matter detection cross-sections. We show the posterior probability distribution function of the spin-independent direct dark matter detection cross-section in Fig. Alc for flat priors in $m_{0}, M_{1 / 2}, \tan \beta$ and $A_{0}$, for $\mu>0$. The most constraining direct detection experiment, XENON-10, can be seen to cover some of the favoured region already, assuming that the flux of dark matter passing through XENON-10 is the same as the galactic average. In

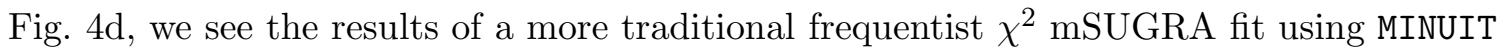
in terms of the lightest CP-even Higgs mass of the MSSM [54]. For each value of the lightest Higgs mass, a $\chi^{2}$ minimisation was performed against all of the other mSUGRA 

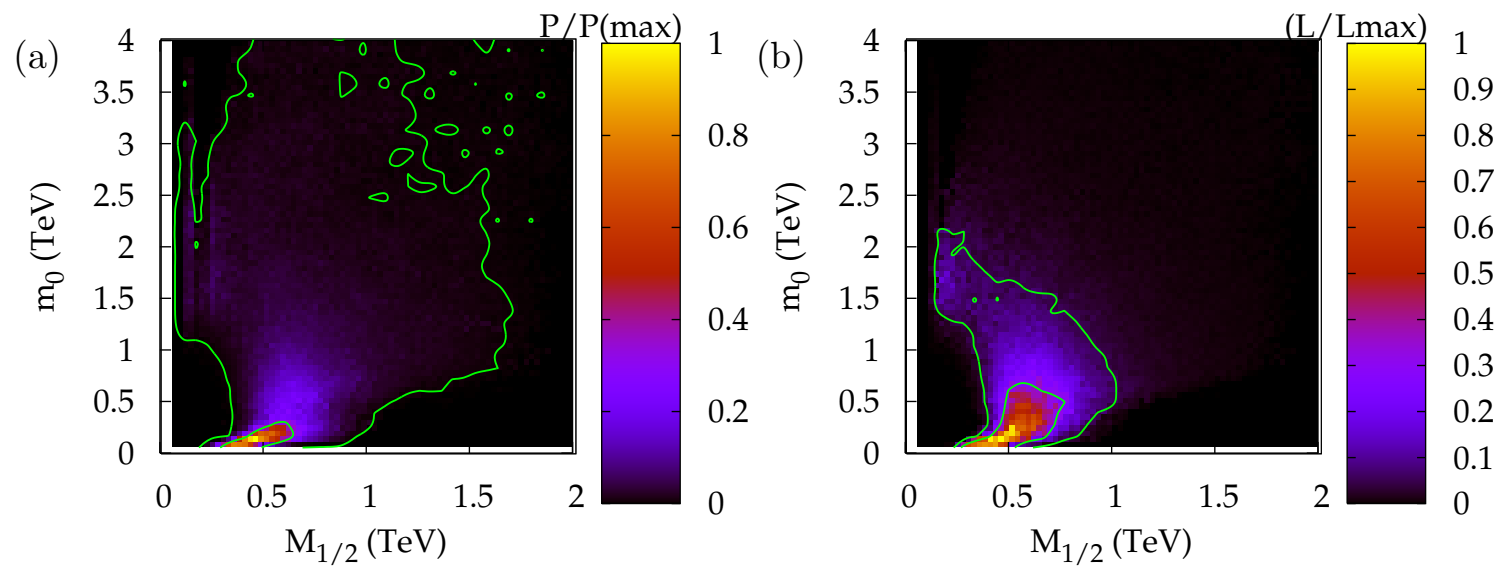

(c)

(d)
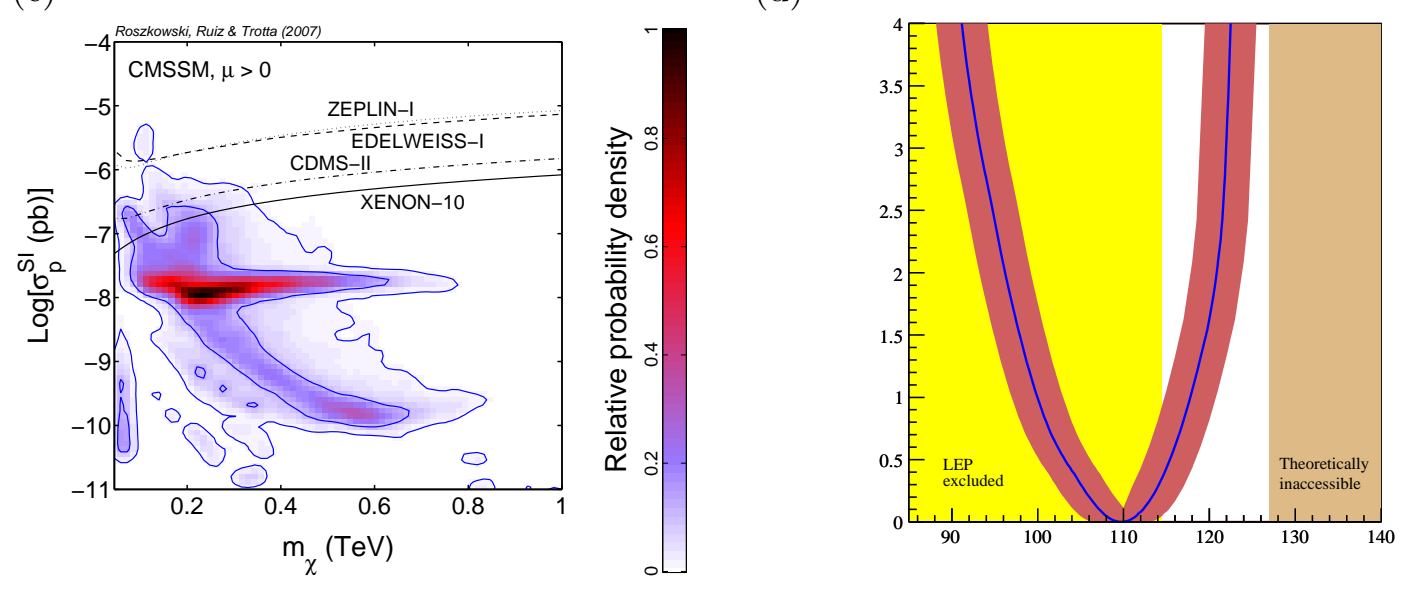

Figure 4: Global mSUGRA fits in the $m_{0}-M_{1 / 2}$ plane: (a) shows the Bayesian posterior probability distribution [59], (b) shows the frequentist interpretation in the same plane [59], (c) displays the direct spin independent detection cross section posterior probability distribution function versus mass of the lightest neutralino for $\mu>0$ along with some $95 \%$ C.L. exclusion contours from direct detection experiments [64]. Inner and outer contours show the $68 \%$ and $95 \%$ confidence level regions respectively. (d) shows the $\Delta \chi^{2}$ of the lightest CP-even Higgs mass from all constraints except for the direct LEP2 Higgs mass constraint [54].

parameters. Many additional electroweak and $b$ observables were included in the $\chi^{2}$ of this fit, although for comparative purposes the LEP2 direct bound on the Higgs mass was left out. This bound is plotted as the yellow excluded region in Fig. $9 \mathrm{~d}$. It can be seen that the global $\chi^{2}$ minimum occurs at $m_{h} \approx 110 \mathrm{GeV}$, below the direct $95 \%$ C.L. lower bound of $114.4 \mathrm{GeV}$. The authors of Ref. [54] use the $\chi^{2}$ curve to infer that $m_{h}=110_{-10}^{+8} \pm 3 \mathrm{GeV}$, where the first uncertainty is statistical and the second uncertainty is theoretical.

\subsection{Data archival}

The samples from MCMC fits take some effort and CPU time to obtain. In principle, the 
mSUGRA fits could be useful to other physicists, who wish to make their own inferences about observables. If a new calculation of SUSY contributions to some observable were to come on-line, statistical inference could be made by simply obtaining some independent samples from the MCMC chains and calculating the new observable for each. A few thousand points might suffice in terms of statistics. Then, the totality of current empirical knowledge about SUSY corrections to the observable is obtained without a need for complicated multi-dimensional fitting procedures. Aside from that, other physicists might be interested in using the chains for their own scans over the points in parameter space that are compatible with current data. For this reason, the authors of Ref. [59] have formed the KISMET web-site, which contains links to text files of the chain data. The weight of each point (the number of times it was visited in the MCMC procedure), along with the values of input parameters, resulting indirect observables, sparticle masses and the likelihood, are listed in the files. Also, 10000 independent samplings from the chains in SLHA format are available from the web-site.

The SuperBayes [52] data are now available to some extent on-line: one can fill out a web-form in order to automatically receive plots of the posterior probability density in dimensions specified by the user 65]. These dimensions can be specified to be observables, input parameters or sparticle masses.

\section{Summary}

Currently, little is empirically known about supersymmetry except for a few indirect data. This tends to lead to under-constrained SUSY models and consequent degeneracies in global fits. However, if the LHC provides some signals that are compatible with SUSY, aside from being an extremely exciting discovery beyond the Standard Model, hypothesis tests against alternative models and even between different classes of SUSY models will be desirable. Ideally, constraints upon the SUSY Lagrangian would be derived with the help of high energy $e^{+} e^{-}$linear collider experiments. A well defined theoretical framework is needed when higher order corrections are included in trying to reconstruct a fundamental SUSY theory and its breaking mechanism. For this purpose, the Supersymmetry Parameter Analysis (SPA) [66] scheme provides a consistent set of conventions and input parameters, as well as a repository for programs which connect parameters in different schemes and relate the Lagrangian parameters to quantities that may be more directly extracted from physical observables such as masses, mixings, decay widths and production cross sections for supersymmetric particles.

There is a somewhat bewildering proliferation of computer program tools for SUSY calculations and phenomenology in the literature. This proliferation is a useful development, and reflects the interest of the high energy physics community in supersymmetry. Even more useful is the collusion, collaboration and organisation between the different programs, to allow results from one to be fed into another program and interpreted automatically. The SUSY Les Houches Accord is a good example of such practice, and it has now become essential for any relevant computer tool to use it so that it can communicate with the other tools. The most popular computer languages that the tools are written in 
are still various versions of fortran and C++. Following the move of most new high energy physics experiments to $\mathrm{C}++$, there is a tendency for new event generators to be written in $\mathrm{C}++$ rather than fortran. In fact the precise language a SUSY tool is written in is becoming less important with the advent of the communication accords, which are in ASCII format. Many of the more sophisticated matrix element or event generators use their own encoding of a Lagrangian to enable the user to define new models. This approach is of obvious use and generality, but packages have many different definitions of the Lagrangian. Perhaps there is a need for yet another accord, so that the same model can easily be fed in to different tools without the need for the user to translate the Lagrangian between the various different conventions.

Finally, we end with a brief summary of the phenomenological SUSY tools that are covered in this review. Since some programs have several different functions, we summarise them all together in Table 2, although the programs are loosely grouped according to their functionality. $\mathcal{L}$ indicates that the tool includes or uses a method of encoding a Lagrangian in order to define extended or new models. In the table, 'Spectrum' indicates that the tool includes an SUSY spectrum calculator, $\nu_{R}$ indicates that RGEs include an option for including right-handed neutrinos (and therefore neutrino mass models), RPV indicates that the tool can handle $R$-parity violation, NMSSM that it can calculate in the Nextto-Minimal Supersymmetric Standard Model, CPV that the tool can take into account complex phases in the SUSY sector and FV that the tool includes some non-minimal flavour violating effects. 'Decays' indicates that the tool automatically calculates the branching ratios of SUSY or SUSY Higgs decays in the MSSM or extensions. Tools which have a positive entry under 'Decay spin' include angular correlation effects from sparticle spins when simulating decays down cascade decay chains. 'ME' indicates a matrix element generator: the code can simulate scattering for $2 \rightarrow N$ hard particles, where $N>2$. 'Initial pol' shows that polarisations of the colliding particles can be taken into account: usually in $e^{+} e^{-}$collisions, but sometimes also in $\gamma \gamma$ or $e \gamma$ collisions. A tick under the $\sigma_{S U S Y}$ heading means that the code has an easy user interface for calculating total or differential crosssections for the production of (sometimes specified) sparticles and/or SUSY Higgs. $e^{+} e^{-}$ and $p p$ indicates that the initial colliding particles can be leptonic or hadronic, respectively. 'Events' mean that individual events are simulated, PS/Had that the program can perform parton showering and/or hadronisation of partons. A tick under the $\Omega_{D M} h^{2}$ header means that the relic density of dark matter can be calculated, $\sigma_{S I, S D}^{p}$ that an estimate of dark matter direct detection is included and 'Ind. DM' that some indirect dark matter detection fluxes are provided. For $b$-observables, $b \rightarrow s \gamma, B \rightarrow \tau \nu, \tau \tau$ and $B_{s} \rightarrow \mu^{+} \mu^{-}$indicates that there is a calculation of the relevant branching ratio including some SUSY effects. A positive entry for $\Delta_{0-}$ means that the program calculates the isospin asymmetry in $B$ decays, whereas an entry under $\Delta M_{B_{s}}$ that the SUSY contributions to $B_{s, d}^{0}-\bar{B}_{s, d}^{0}$ mixing are calculated. A $(g-2)_{\mu}$ entry indicates that a SUSY contribution to the anomalous magnetic moment of the muon can be easily extracted from the tool, whereas EW means that some electroweak observables are provided: usually $\Delta \rho$ and $M_{W}$. A tick under edm means that electric dipole moments can be calculated, whereas 'Fits' indicates a fitting tool that can fit either collider observables and/or indirect constraints such as EW observables 
and dark matter relic densities. An entry under 'Web form' gives the reference including a link to a web-form where results from the program can be automatically obtained by filling in a form on the world-wide web. Finally the 'code' column indicates that the package can output computer code, which can then be compiled into a numerical program in order to evaluate observables. Prospective users are warned that multi-functionality does not necessarily mean a more accurate calculation and indeed in some cases, the converse will apply. It is hoped that Table 2 will help point prospective new users towards the SUSY tool(s) that they require.

\section{Acknowledgments}

This review has been partially supported by the STFC. A much shortened form was originally conceived for a talk in the SUSY 07 conference. It borrows heavily from the manuals and web-pages of the tools discussed, the BSM tools repository [8] and the SUSY Les Houches Accord web-pages [1]. We thank G Bélanger, F Boudjema, T Hahn, S Heinemeyer, J S Lee, N Mahmoudi, F Maltoni, T Plehn, W Porod, P Richardson, S Schumann, $\mathrm{P}$ Slavich and B Webber for corrections and suggestions on the draft.

\section{References}

[1] P. Skands, B.C. Allanach et al, The SUSY Les Houches Accord: Interfacing SUSY Spectrum Calculators, Decay Packages and Event Generators, JHEP 0407 (2004) 036, [arXiv:hep-ph/0311123]. http://home.fnal.gov/ ${ }^{\sim}$ skands/slha/

[2] T. Hahn, SUSY Les Houches Accord I/O made easy, arXiv:hep-ph/0408283; T. Hahn, SUSY Les Houches Accord 2 I/O made easy, arXiv:hep-ph/0605049. http://www.feynarts.de/slha

[3] B.C. Allanach et al, Susy Les Houches Accord 2, [arXiv:0801.0045]; ibid, The SUSY Les Houches Accord Conventions, in Physics Beyond the Standard Model: Supersymmetry, proceedings of the 2007 Les Houches "Physics at TeV Colliders" Workshop, [arXiv:0802.3672]. http://home.fnal.gov/ skands/slha/

[4] E. Boos et al, Generic user process interface for event generators, in Proceedings of the Workshop on Physics at TeV Colliders II Workshop, [arXiv:0109068].

[5] J. Alwall et al., A standard format for Les Houches event files, Comput. Phys. Commun. 176 (2007) 300 [arXiv:hep-ph/0609017].

[6] J. Alwall et al., A Les Houches Interface for BSM Generators, arXiv:0712.3311 [hep-ph].

[7] P. Z. Skands, A quick guide to SUSY tools, arXiv:hep-ph/0601103.

[8] P. Z. Skands et al., A repository for beyond-the-standard-model tools, in BSM Working Group summary report of Les Houches at TeV Colliders 2005, arXiv:hep-ph/0602198. http://www.ippp.dur.ac.uk/montecarlo/BSM/

[9] F. E. Paige, S. D. Protopopescu, H. Baer and X. Tata, ISAJET 7.69: A Monte Carlo event generator for $p$, anti-p $p$, and e+ $e$-reactions, arXiv:hep-ph/0312045. http://www.hep.fsu.edu/ isajet/ 
[10] C. Balazs, see ISALHA.F, ISALHD.F and LHAISA.F links on the SLHA page http://home.fnal.gov/ $\sim$ skands/slha/.

[11] B.C. Allanach, SOFTSUSY: A program for calculating supersymmetric spectra, Comput. Phys. Commun. 143 (2002) 305, [arXiv:hep-ph/0104145]. http://projects.hepforge.org/softsusy/.

[12] W. Porod, SPheno, a program for calculating supersymmetric spectra, SUSY particle decays and SUSY particle production at e+ e- colliders, Comput. Phys. Commun. 153 (2003) 275 [arXiv:hep-ph/0301101]. http://ific.uv.es/ porod/SPheno.html

[13] A. Djouadi, J. L. Kneur and G. Moultaka, SuSpect: A Fortran code for the supersymmetric and Higgs particle spectrum in the MSSM, Comput. Phys. Commun. 176 (2007) 426 [arXiv:hep-ph/0211331]. http://www.lpta.univ-montp2.fr/users/kneur/Suspect/

[14] S. Kraml, Comparison of SUSY spectrum generators: mass spectra, relic densities, etc, http://kraml.home.cern.ch/kraml/comparison/compare.htm]

[15] B.C. Allanach, S. Kraml and W. Porod, Theoretical uncertainties in sparticle mass predictions from computational tools, JHEP 03 (2003) 016, hep-ph/0302102; B. C. Allanach, A. Djouadi, J. L. Kneur, W. Porod and P. Slavich, Precise determination of the neutral Higgs boson masses in the MSSM, JHEP 0409 (2004) 044 [arXiv:hep-ph/0406166]. G. Belanger, $\mathrm{S}$. Kraml and A. Pukhov, Comparison of SUSY spectrum calculations and impact on the relic density constraints from WMAP, Phys. Rev. D 72 (2005) 015003 [arXiv:hep-ph/0502079].

[16] M. Bahr et al., Herwig++ Physics and Manual, arXiv:0803.0883 [hep-ph]. http://projects.hepforge.org/herwig/

[17] J. Alwall et al., MadGraph/MadEvent v4: The New Web Generation, JHEP 0709 (2007) 028 [arXiv:0706.2334 [hep-ph]]; G. C. Cho, K. Hagiwara, J. Kanzaki, T. Plehn, D. Rainwater and T. Stelzer, Weak boson fusion production of supersymmetric particles at the LHC, Phys. Rev. D 73 (2006) 054002 [arXiv:hep-ph/0601063]. http://cp3wks05.fynu.ucl.ac.be/twiki/bin/view/Main/WebHome

[18] T. Sjostrand, S. Mrenna and P. Skands, PYTHIA 6.4 physics and manual, JHEP 0605 (2006) 026 [arXiv:hep-ph/0603175]. http://home.thep.lu.se/ ${ }^{\sim}$ torbjorn/Pythia.htm

[19] M. Muhlleitner, A. Djouadi and Y. Mambrini, SDECAY: A Fortran code for the decays of the supersymmetric particles in the MSSM, Comput. Phys. Commun. 168 (2005) 46 [arXiv:hep-ph/0311167]. http://lappweb.in2p3.fr/ ${ }^{\sim}$ muehlleitner/SDECAY/

[20] P. Richardson, Spin correlations in Monte Carlo simulations, JHEP 0111 (2001) 029 [arXiv:hep-ph/0110108].

[21] P. Meade and M. Reece, BRIDGE: Branching ratio inquiry / decay generated events, arXiv:hep-ph/0703031. http://www.lepp.cornell.edu/public/theory/BRIDGE/

[22] S. Heinemeyer, W. Hollik and G. Weiglein, FeynHiggs: A program for the calculation of the masses of the neutral CP-even Higgs bosons in the MSSM, Comput. Phys. Commun. 124 (2000) 76 [arXiv:hep-ph/9812320]; S. Heinemeyer, W. Hollik and G. Weiglein, The masses of 
the neutral CP-even Higgs bosons in the MSSM: Accurate analysis at the two-loop level, Eur. Phys. J. C 9 (1999) 343 [arXiv:hep-ph/9812472]; G. Degrassi, S. Heinemeyer, W. Hollik, P. Slavich and G. Weiglein, Towards high-precision predictions for the MSSM Higgs sector, Eur. Phys. J. C 28 (2003) 133 [arXiv:hep-ph/0212020]; M. Frank, T. Hahn, S. Heinemeyer, W. Hollik, H. Rzehak and G. Weiglein, The Higgs boson masses and mixings of the complex MSSM in the Feynman-diagrammatic approach, JHEP 0702 (2007) 047

[arXiv:hep-ph/0611326].

http://www.feynhiggs.de/

[23] J. S. Lee, M. Carena, J. Ellis, A. Pilaftsis and C. E. M. Wagner, CPsuperH2.0: an Improved Computational Tool for Higgs Phenomenology in the MSSM with Explicit CP Violation, arXiv:0712.2360 [hep-ph]; J. S. Lee, A. Pilaftsis, M. S. Carena, S. Y. Choi, M. Drees, J. R. Ellis and C. E. M. Wagner, CPsuperH: A computational tool for Higgs phenomenology in the minimal supersymmetric standard model with explicit $C P$ violation, Comput. Phys. Commun. 156 (2004) 283 [arXiv:hep-ph/0307377]. http://www.hep.man.ac.uk/u/jslee/CPsuperH.htm]

[24] A. Djouadi, J. Kalinowski and M. Spira, HDECAY: A program for Higgs boson decays in the standard model and its supersymmetric extension, Comput. Phys. Commun. 108 (1998) 56 [arXiv:hep-ph/9704448]. http://people.web.psi.ch/spira/hdecay/

[25] S. Béjar and J. Guasch. http://fchdecay.googlepages.com/

[26] U. Ellwanger and C. Hugonie, NMSPEC: A Fortran code for the sparticle and Higgs masses in the NMSSM with GUT scale boundary conditions, Comput. Phys. Commun. 177 (2007) 399 [arXiv:hep-ph/0612134]; U. Ellwanger, J. F. Gunion and C. Hugonie, NMHDECAY: A Fortran code for the Higgs masses, couplings and decay widths in the NMSSM, JHEP 0502 (2005) 066 [arXiv:hep-ph/0406215]. http://www.th.u-psud.fr/NMHDECAY/nmssmtools.htm

[27] T. Hahn and M. Perez-Victoria, Automatized one-loop calculations in four and D dimensions, Comput. Phys. Commun. 118 (1999) 153 [arXiv:hep-ph/9807565]; T. Hahn, Generating Feynman diagrams and amplitudes with FeynArts 3, Comput. Phys. Commun. 140 (2001) 418 [arXiv:hep-ph/0012260]; T. Hahn and C. Schappacher, The implementation of the minimal supersymmetric standard model in FeynArts and FormCalc, Comput. Phys. Commun. 143 (2002) 54 [arXiv:hep-ph/0105349]. http://www.feynarts.de/

[28] M. Rauch, Quantum Effects in Higgs-Boson Production Processes at Hadron Colliders, arXiv:0804.2428 [hep-ph]. http://www.ph.ed.ac.uk/ mrauch/HadCalc/

[29] A. Pukhov et al., CompHEP: A package for evaluation of Feynman diagrams and integration over multi-particle phase space. User's manual for version 33, arXiv:hep-ph/9908288; A. Pukhov, CalcHEP 3.2: MSSM, structure functions, event generation, batchs, and generation of matrix elements for other packages, arXiv:hep-ph/0412191. http://www.ifh.de/ pukhov/calchep.htm

[30] E. Boos et al. [CompHEP Collaboration], CompHEP 4.4: Automatic computations from Lagrangians to events, Nucl. Instrum. Meth. A 534 (2004) 250 [arXiv:hep-ph/0403113] and Ref. [29]. http://comphep.sinp.msu.ru 
[31] A. V. Semenov, LanHEP: A package for automatic generation of Feynman rules in field theory. Version 2.0, arXiv:hep-ph/0208011. http://theory.sinp.msu.ru/ $\sim$ semenov/lanhep.htm]

[32] H. Murayama, I. Watanabe and K. Hagiwara, Evaluating cross-sections at TeV energy scale by HELAS, Tsukuba Workshop JLC 1992265. http://www.pas.rochester.edu/ rain/smadgraph/HELAS.ps.g

[33] T. Gleisberg, S. Hoche, F. Krauss, A. Schalicke, S. Schumann and J. C. Winter, SHERPA 1.alpha, a proof-of-concept version, JHEP 0402 (2004) 056 [arXiv:hep-ph/0311263]. http://www.sherpa-mc.de/

[34] F. Krauss, R. Kuhn and G. Soff, AMEGIC ++ 1.0: A matrix element generator in $C++$, JHEP 0202 (2002) 044 [arXiv:hep-ph/0109036].

[35] W. Kilian, T. Ohl and J. Reuter, WHIZARD: Simulating Multi-Particle Processes at LHC and ILC, arXiv:0708.4233 [hep-ph]. http://whizard.event-generator.org/

[36] M. Moretti, T. Ohl and J. Reuter, O'Mega: An optimizing matrix element generator, arXiv:hep-ph/0102195.

[37] N. Ghodbane, SUSYGEN3: An event generator for linear colliders, arXiv:hep-ph/9909499. http://lyoinfo.in2p3.fr/susygen/susygen3.htm

[38] J. Fujimoto et al., GRACE/SUSY: Automatic generation of tree amplitudes in the minimal supersymmetric standard model, Comput. Phys. Commun. 153 (2003) 106 [arXiv:hep-ph/0208036]. http://minami-home.kek.jp/

[39] K. Hagiwara et al., Supersymmetry simulations with off-shell effects for LHC and ILC, Phys. Rev. D 73 (2006) 055005 [arXiv:hep-ph/0512260].

[40] W. Beenakker, R. Hopker, M. Spira and P. M. Zerwas, Squark and gluino production at hadron colliders, Nucl. Phys. B 492 (1997) 51 [arXiv:hep-ph/9610490]. http://www.ph.ed.ac.uk/ tplehn/prospino/

[41] A. Freitas, A. von Manteuffel and P. M. Zerwas, Slepton production at $e+e$ - and $e$ - $e$ - linear colliders, Eur. Phys. J. C 34 (2004) 487 [arXiv:hep-ph/0310182]. http://theory.fnal.gov/people/freitas/

[42] M. A. Dobbs et al., Les Houches guidebook to Monte Carlo generators for hadron collider physics, arXiv:hep-ph/0403045.

[43] L. Lönnbladd, ThePEG Reference Manual. http://projects.hepforge.org/thepeg/doxygen/index.htm

[44] S. Catani, F. Krauss, R. Kuhn and B. R. Webber, QCD matrix elements + parton showers, JHEP 0111 (2001) 063 [arXiv:hep-ph/0109231].

[45] J. C. Winter, F. Krauss and G. Soff, A modified cluster-hadronization model, Eur. Phys. J. C 36 (2004) 381 [arXiv:hep-ph/0311085].

[46] G. Hinshaw et al. [WMAP Collaboration], Five-Year Wilkinson Microwave Anisotropy Probe (WMAP) Observations:Data Processing, Sky Maps, $\mathcal{E}$ Basic Results, arXiv:0803.0732 [astro-ph]. 
[47] F. Boudjema, A. Semenov and D. Temes, SUSY dark matter: Loops and precision from particle physics, Nucl. Phys. Proc. Suppl. 157 (2006) 172; N. Baro, F. Boudjema and A. Semenov, Full one-loop corrections to the relic density in the MSSM: A few examples, Phys. Lett. B 660 (2008) 550 [arXiv:0710.1821 [hep-ph]].

[48] P. Gondolo, J. Edsjo, P. Ullio, L. Bergstrom, M. Schelke and E. A. Baltz, DarkSUSY: Computing supersymmetric dark matter properties numerically, JCAP 0407 (2004) 008 [arXiv:astro-ph/0406204]. http://www.physto.se/ ${ }^{2}$ edsjo/darksusy

[49] G. Belanger, F. Boudjema, A. Pukhov and A. Semenov, Dark matter direct detection rate in a generic model with micrOMEGAs2.1, arXiv:0803.2360 [hep-ph]; G. Belanger, F. Boudjema, A. Pukhov and A. Semenov, micrOMEGAs2.0: A program to calculate the relic density of dark matter in a generic model, Comput. Phys. Commun. 176 (2007) 367 [arXiv:hep-ph/0607059]. http://wwwlapp.in2p3.fr/lapth/micromegas/

[50] G. Degrassi, P. Gambino and P. Slavich, SusyBSG: a fortran code for BR[B $\rightarrow$ Xs gamma] in the MSSM with Minimal Flavor Violation, arXiv:0712.3265 [hep-ph]. http://slavich.web.cern.ch/slavich/susybsg/home.html

[51] F. Mahmoudi, SuperIso: A program for calculating the isospin asymmetry of $B \rightarrow K^{*} \gamma$ in the MSSM, Comput. Phys. Commun. 178 (2008) 745 [arXiv:0710.2067 [hep-ph]]. http://www.isv.uu.se/ nazila/superiso/

[52] R. Ruiz de Austri and R. Trotta, SuperBayeS Supersymmetry Parameters Extraction Routines for Bayesian Statistics. http://superbayes.org/

[53] F. James, MINUIT: Function minimization and error analysis, CERN Program Library Long Writeup D506, http://wwwasdoc.web.cern.ch/wwwasdoc/minuit/minmain.htm

[54] O. Buchmueller et al., Prediction for the Lightest Higgs Boson Mass in the CMSSM using Indirect Experimental Constraints, Phys. Lett. B 657 (2007) 87 [arXiv:0707.3447 [hep-ph]].

[55] R. Trotta, Bayes in the sky: Bayesian inference and model selection in cosmology, arXiv:0803.4089 [astro-ph].

[56] A. Lewis and S. Bridle, Cosmological parameters from CMB and other data: a Monte-Carlo approach, Phys. Rev. D66 (2002) 103511 [arXiv:astro-ph/0205436]. http://cosmologist.info/cosmom

[57] N. Metropolis, A.W. Rosenbluth, M.N. Teller and E. Teller, Equations of State Calculations by Fast Computing Machines, Journal of Chemical Physics, 21 (1953) 1087-1091

[58] A. Gelman and D. Rubin, Inference from Iterative Simulation Using Multiple Sequences, Stat. Sci. 7 (1992) 457.

[59] B.C. Allanach, K. Cranmer, C.G. Lester and A.M. Weber, Natural priors, CMSSM fits and LHC weather forecasts, JHEP 0708 (2007) 023 [arXiv:0705.0487]. http://users.hepforge.org/ allanach/benchmarks/kismet.htm

[60] R. Lafaye, T. Plehn, M. Rauch and D. Zerwas, Measuring Supersymmetry, arXiv:0709.3985 [hep-ph]. 
[61] B.C. Allanach and C.G. Lester, Sampling using a 'bank' of clues, arXiv:0705.0486.

[62] P. Bechtle, K. Desch and P. Wienemann, Fittino, a program for determining MSSM parameters from collider observables using an iterative method, Comput. Phys. Commun. $\mathbf{1 7 4}$ (2006) 47 [arXiv:hep-ph/0412012]. http://www-flc.desy.de/fittino/

[63] F. Feroz and M. P. Hobson, Multimodal nested sampling: an efficient and robust alternative to MCMC methods for astronomical data analysis, arXiv:0704.3704 [astro-ph].

[64] L. Roszkowski, R. Ruiz de Austri and R. Trotta, Implications for the Constrained MSSM from a new prediction for $b$ to s gamma, JHEP 0707 (2007) 075 [arXiv:0705.2012 [hep-ph]].

[65] Dark Matter Network Exclusion Program. http://pisrv0.pit.physik.uni-tuebingen.de/darkmatter/

[66] J. A. Aguilar-Saavedra et al., Supersymmetry parameter analysis: SPA convention and project, Eur. Phys. J. C 46 (2006) 43 [arXiv:hep-ph/0511344]. 


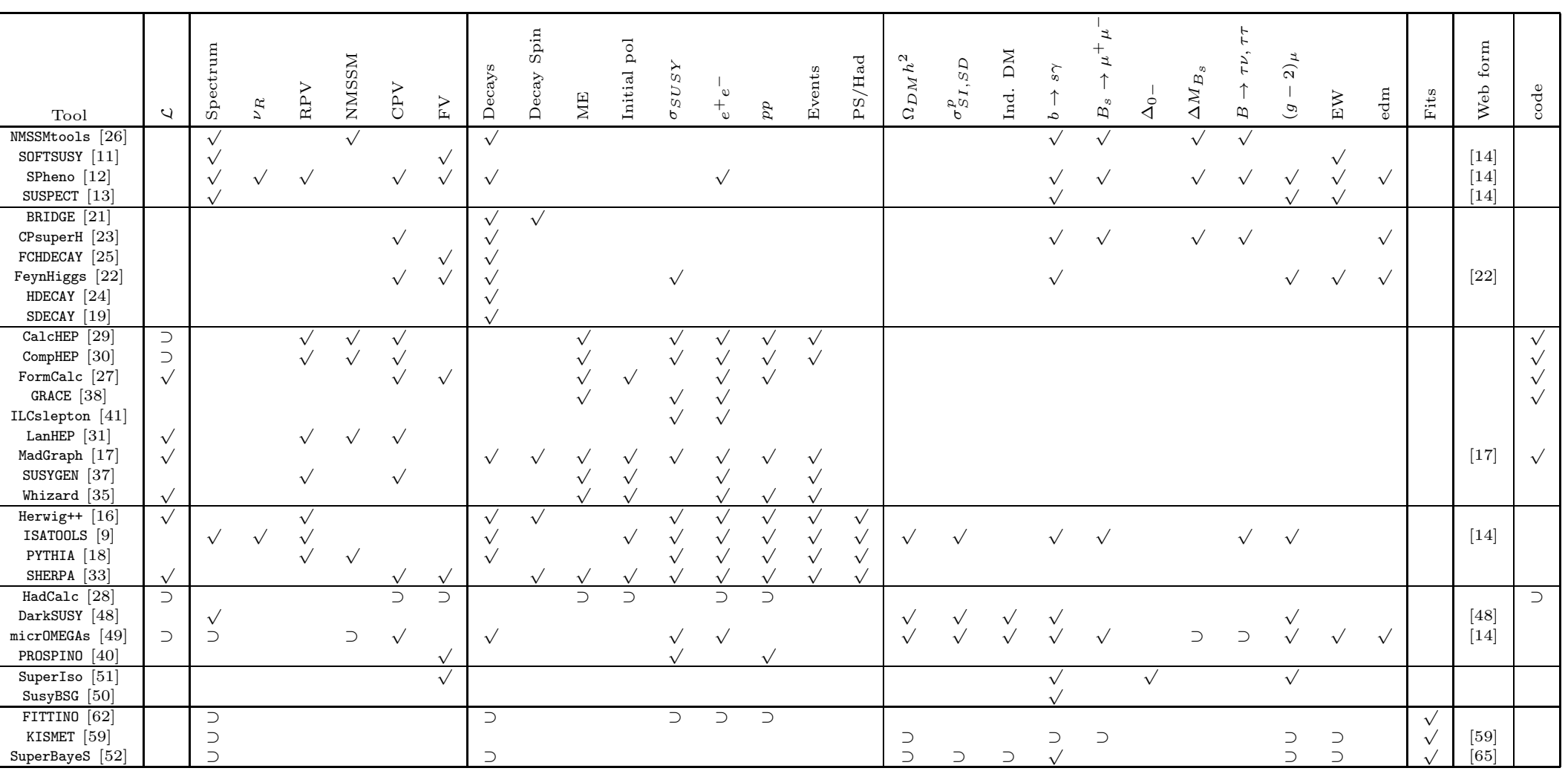

Table 2: Summary of functionality of current, publicly available, supported SUSY tools. A $\sqrt{ }$ indicates that there is some support for the feature in question, but makes no claims about the accuracy of the calculation. $\supset$ indicates that the one of the other packages in the table is included in the distribution in order to provide the relevant functionality. See section 8 for a description of the various features. 